\title{
Metabolic effects of adenosine on regional myocardial ischemia by phosphorus 31 nuclear magnetic resonance spectroscopy
}

\begin{abstract}
The metabolic effects of adenosine on regionally ischemic myocardium were investigated in an open-chest rabbit model by means of phosphorus 31 nuclear magnetic resonance (NMR) spectroscopy. Sixteen anesthetized New Zealand white rabbits were subjected to thoracotomy; a reversible snare occluder was placed around a large branch of the left circumflex coronary artery, and an NMR surface coil was positioned adjacent to the myocardium perfused by this vessel. The animals were placed in a $2.0 \mathrm{~T} \mathrm{CSI} \mathrm{spectrometer} \mathrm{(GE} \mathrm{Medical} \mathrm{Systems,} \mathrm{Fremont,}$ Calif.), and baseline spectra were acquired. Eight animals were treated with intravenous adenosine (25 $\mathrm{mg} / \mathrm{kg})$, and eight rabbits served as control subjects. All animals were subjected to a 10-minute period of ischemia followed by a period of reperfusion. NMR spectra were acquired during both intervals. During the occlusion period, expected increases in inorganic phosphate levels and decreases in phosphocreatine levels were observed in both groups; however, inorganic phosphate increased less in adenosine-treated animals (adenosine: $33 \pm 2.8 \%$ total spectral area during occlusion vs control: $41 \pm 3.1 \%$ ) and phosphocreatine diminished less with adenosine (adenosine: $26 \pm 3 \%$ vs control: $13 \pm 1.2 \% ; p<0.002$ ). No significant differences were seen in $\beta$-adenosine triphosphate levels. In both groups the metabolite levels during reperfusion recovered to near baseline values, although phosphocreatine remained slightly higher in the treated group during early reperfusion. An apparent cardioprotective effect of adenosine on relative phosphocreatine and inorganic phosphate levels can be observed in intact rabbits by means of phosphorus 31 NMR spectroscopy. (AM HEART J 1992;124:1205.)
\end{abstract}

Pamela M. Marcovitz, MD, ${ }^{a}$ Alex M. Aisen, MD, ${ }^{b}$ Laura E. Fencil, MD, PhD, ${ }^{b}$ Scott D. Swanson, $\mathrm{PhD},{ }^{b}$ and Andrew J. Buda, $\mathrm{MD}^{\mathrm{a}, \mathrm{c}}$ Ann Arbor, Mich., and New Orleans, La.

Depletion of high-energy phosphates is a well-known phenomenon that occurs during experimental myocardial ischemia and reperfusion. ${ }^{1}$ In studies correlating levels of high-energy phosphates with myocardial contractility, a loss of high-energy phosphates (phosphocreatine and adenosine triphosphate) during ischemia, along with a concomitant increase in inorganic phosphate levels, is associated with loss of contractile function..$^{2,3}$ On the other hand, relative

\footnotetext{
From the Departments of anternal Medicine (Division of Cardiology); ${ }^{b}$ Radiology, University of Michigan Medical Center; and ${ }^{\mathrm{c}}$ Department of Medicine (Cardiology Section), Tulane University Medical Center, New Orleans.

Supported in part by National Institutes of Health grant R01 HL-34691 (Dr. Buda)

Received for publication March 16, 1992; accepted May 1, 1992.

Reprint requests: Andrew Buda, MD, Cardiology Section, Department of Medicine, Tulane University Medical Center, 1430 Tulane Ave., New Orleans, LA 70112-2699.

$4 / 1 / 40453$
}

preservation of adenosine triphosphate (A'TP) during ischemia and reperfusion is associated with reversible myocardial injury. ${ }^{4}$ Phosphorus 31 nuclear magnetic resonance (NMR) spectroscopy is a nontissue-destructive method for measuring the relative concentrations of high-energy phosphates in intact myocardium during ischemia and reperfusion. Results of previous studies in intact animals with the use of NMR spectroscopy have demonstrated the expected changes in high-energy phosphate metabolism during ischemia and reperfusion, including a profound depletion of phosphocreatine, a decrease in ATP, and a concomitant increase in inorganic phosphate with a return toward baseline of these values on reperfusion of viable myocardium. ${ }^{5-7}{ }^{31} \mathrm{P}$ NMR studies can thus provide information regarding cell viability during ischemia and reperfusion and can help assess the effects of various interventions on ischemic and reperfused myocardium. $4,8,9$

Adenosine has been shown to reduce myocardial injury after prolonged periods of ischemia and to 


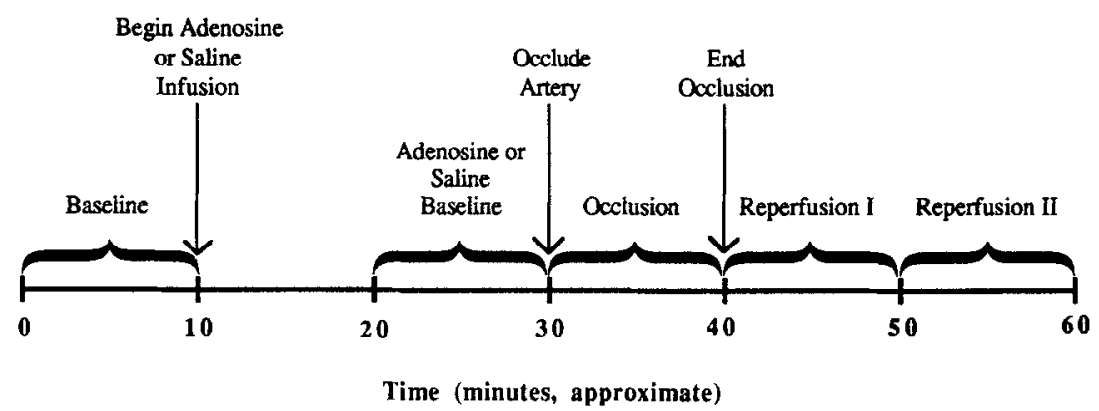

Fig. 1. Time line showing experimental protocol.

limit infarct size. ${ }^{10-12}$ The mechanism by which adenosine exerts its cardioprotective effect is as yet, speculative. The compound is a potent vasodilator and may play a role in autoregulation of coronary artery flow. It also has an indirect negative inotropic effect. ${ }^{13}$ One can speculate that adenosine might play a role in decreasing degradation or increasing repletion of high-energy phosphates through the provision of precursors for ATP synthesis. Finally, the agent may exert a beneficial effect by acting on neutrophils to limit reperfusion injury. ${ }^{14}$

NMR studies to date have examined the cardioprotective effects of adenosine in the isolated perfused heart ${ }^{15}$; however, to our knowledge no ${ }^{31} \mathrm{P}$ NMR studies have examined the protective effects of adenosine in the intact animal model. Because isolated heart preparations may behave differently metabolically from the intact myocardium, it is important to examine the metabolic changes seen during ischemia and reperfusion in intact animals. In addition, previous studies with adenosine in the intact animal nodel, in which biochemical and histopathologic techniques were used, have generally examined its cardioprotective effects during prolonged (90 minutes or more) periods of ischemia. ${ }^{10-12}$ Few studies have considered the actions of adenosine during a brief period of ischemia comparable to that which would result in myocardial stunning. Accordingly this study was undertaken to examine whether adenosine would provide a beneficial effect on highenergy phosphate metabolism as measured by ${ }^{31} \mathrm{P}$ NMR spectroscopy during a brief period of ischemia and reperfusion in intact animals.

\section{METHODS}

Sixteen New Zealand white rabbits weighing 3 to $5 \mathrm{~kg}$ were anesthetized with xylazine $(10 \mathrm{mg} / \mathrm{kg})$ and ketamine $(40 \mathrm{mg} / \mathrm{kg}$ ) via intramuscular injection. The internal jugular vein was cannulated for maintenance of anesthesia with small boluses of pentobarbital $(0.2$ to $0.5 \mathrm{cc})$ and for intravenous infusion of adenosine or normal saline solution. The animals underwent tracheal intubation and were placed on a Harvard rodent ventilator (Harvard Apparatus, Inc., South Natick, Mass.) with a mixture of $95 \%$ oxygen and $5 \%$ carbon dioxide. The anesthetized animals underwent thoracotomy. A reversible snare occluder consisting of 6-0 suture threaded through a small plastic catheter was placed around a large marginal branch of the left circumflex coronary artery. During occlusion the catheter was slid down onto the surface of the artery to produce occlusion and held in place with a plastic clip. A four-turn $13 \mathrm{~mm}$ solenoidal radiofrequency coil was sutured onto the epicardial surface over the region of myocardium to be rendered ischemic. The animals were then placed in the bore of a General Electric 2.0 T CSI spectrometer (GE Medical Systems, Fremont Calif.). Shimming was performed on the proton signal, yielding a line width of three to five parts per million for the water peak. Ten rabbits were studied with ECG gating and six rabbits with respiratory gating, which was found to reduce line-broadening motion artifact. At the onset of the experiment, baseline spectra were obtained over a 10-minute period by averaging 256 spectral acquisitions. After baseline spectra were obtained, an infusion of adenosine $(25 \mathrm{mg} / \mathrm{kg}), 2 \mathrm{mg} / \mathrm{min}$, or normal saline solution was begun. After a 10 -minute equilibration period, a second baseline reading was obtained. The snare was then tightened around the coronary artery to produce occlusion, and another spectrum was obtained. The snare was then loosened to allow reperfusion, and two more spectra were obtained consecutively at 10 -minute intervals. A diagram of the protocol is shown in Fig. 1.

Ten-Hertz line broadening was used to filter the spectrum, and phasing was carried out with a computer program with manual correction performed as needed. The areas under five peaks corresponding to inorganic phosphate, phosphocreatine, $\gamma-, \alpha-$, and $\beta$-ATP were determined by means of a Lorentzian peak-fitting program (NMR1, New Methods Research, Inc., Syracuse, N.Y.). Individual peak areas were expressed as percentages of total peak area of the inorganic phosphate, phosphocreatine, and three ATP peaks. The phosphocreatine:inorganic phosphate ratio was also calculated. $\mathrm{pH}$ was calculated from the shift of inorganic phosphate during ischemia in relation to phosphocreatine as previously described $.^{16} \mathrm{Ex}-$ cept as noted, all data are expressed as mean \pm standard 

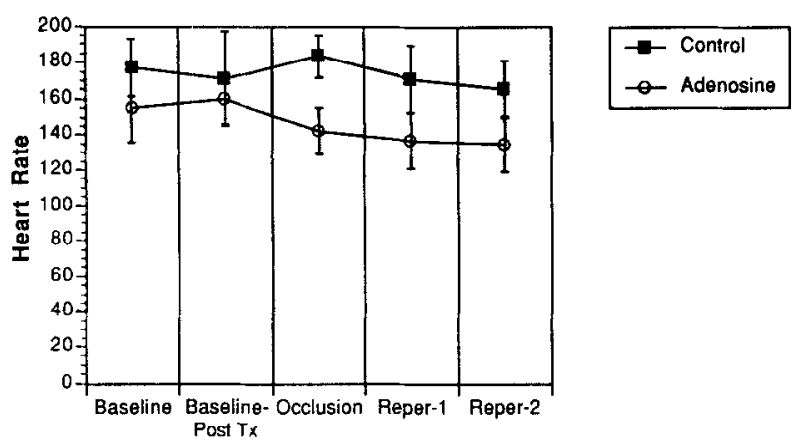

Fig. 2. Mean heart rates of the two groups are shown. Error bars are standard deviations. For technical reasons, heart rates were not obtained from all animals, particularly those animals in which respiratory rather than ECG gating was used ( $n=5$ for control group; $n=6$ for treatment group).

error of the mean. Statistical analysis was performed with unpaired Student's $t$ test.

\section{RESULTS}

Heart rates are shown in Fig. 2. As noted, rate data were not collected from all animals for technical reasons, particularly when respiratory rather than cardiac gating was used. The heart rate decreased slightly in adenosine-treated animals during the occlusion and reperfusion periods, and averaged approximately $20 \%$ lower than in the control group.

Representative spectra are shown in Fig. 3 . The $\mathrm{pH}$ measured during coronary occlusion did not differ between adenosine-treated and control animals (Fig. 4). The $\mathrm{pH}$ was not measured during reperfusion, since it was difficult to accurately measure the shift in inorganic phosphate resulting from the small size and spectral contamination of the inorganic phosphate peak, which probably contains contributions from phospho-, mono-, and diesters, as well as 2,3-diphosphoglycerate (DPG).

Levels of phosphocreatine decreased significantly with occlusion in both groups (Fig. 5). In the adenosine-treated group, however, the decrease was much less pronounced than in the control group (phosphocreatine levels during occlusion were $26 \pm 3 \%$ in the treatment group compared with $13 \pm 1.2 \%$ in the control group, $p<0.002$ ). With reperfusion, phosphocreatine values returned to baseline in both groups but tended to remain higher in the adenosinetreated group during the first reperfusion period $(38 \pm 3.2 \%$ as compared with $30 \pm 2.5 \% ; p<0.10$ ). By the second reperfusion period, there were no significant differences between the adenosine-treated and control groups.

Inorganic phosphate levels increased significantly

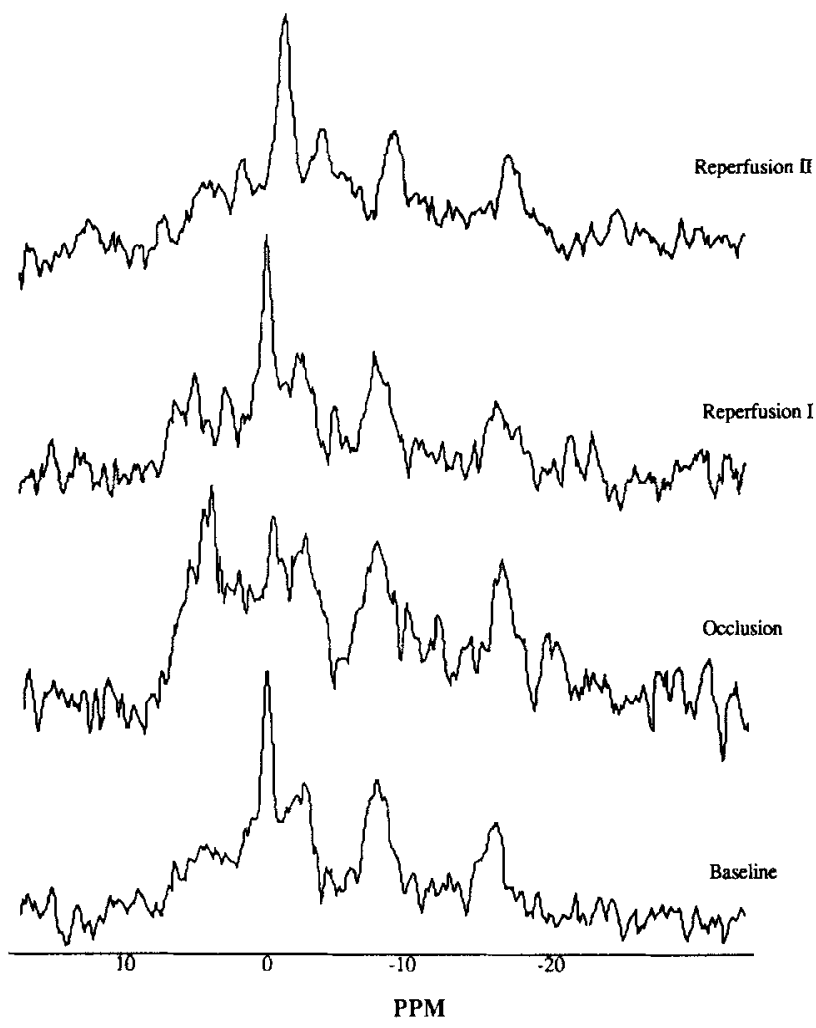

Fig. 3. Representative spectra obtained during baseline, occlusion, and reperfusion periods. Spectra are shown after phasing and baseline correction.

during occlusion in both groups; however, the magnitude of this increase during occlusion was less in the adenosine-treated group than in the control group ( $33 \pm 2.8 \%$ for treated animals vs $41 \pm 3.1 \%$ for control animals; $p=0.06$ ). Both returned to normal during reperfusion, and there were no significant differences in inorganic phosphate levels by the end of reperfusion (Fig. 6).

Fig. 7 depicts $\beta$-ATP levels during baseline, occlusion, and both reperfusion periods. There were no significant differences between the treated and nontreated groups with regard to $\beta$-ATP levels during occlusion and reperfusion. The phosphocreatine: inorganic phosphate ratio, an indicator of mitochondrial function, was calculated at baseline, occlusion, and both reperfusion periods (Fig. 8). This ratio was significantly higher in the adenosine-treated group than in the control group during occlusion, with evidence of a similar trend during reperfusion.

\section{DISCUSSION}

In this investigation, decreases in high-energy phosphate, phosphocreatine, and ATP levels were observed during a brief reversible period of regional myocardial ischemia, with a return toward baseline 

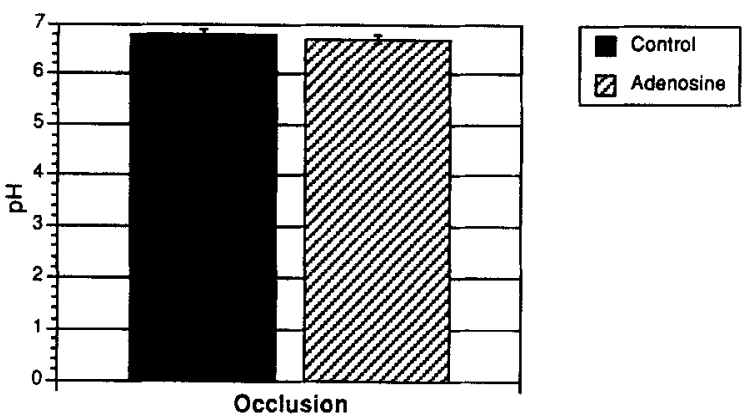

Fig. 4. $\mathrm{pH}$ data during occlusion are shown (mean \pm SEM).

values of these compounds during reperfusion. The depletion of phosphocreatine and ATP was less pronounced in the adenosine-treated group compared with the control group. A concomitant increase in inorganic phosphate levels was seen with occlusion in both groups but was of a greater magnitude in the control group. Although the effects of adenosine on high-energy phosphate levels was most evident during ischemia, there was a trend toward increased phosphocreatine levels in the adenosine-treated group during early reperfusion. We note that our measurements represent an average of signal from all myocardium proximate to the coil. Hence the observations may be a result of differences in both the size of the ischemic zone and differences in high-energy phosphate levels within the ischemic tissue.

There are several possible mechanisms for these apparent cardioprotective effects. These include increased coronary arterial flow, reduced myocardial oxygen demand as a result of the negative inotropic effects of adenosine, inhibition of reperfusion injury, and possibly a metabolic role in improved ATP synthesis or decreasing ATP degradation consequent to the role of adenosine as a metabolic intermediate. The present study does not allow us to distinguish among these possible mechanisms.

Previous reports have demonstrated beneficial effects of adenosine on the myocardium during reperfusion after ischemic injury. In the majority of these studies, the compound was administered during reperfusion. Olaffson et al. ${ }^{10}$ observed a significant reduction in infarct size in adenosine-treated animals after a 90-minute period of coronary occlusion, with preservation of endothelial structure and less neutrophilic infiltration in the subendocardial regions of injured zones. Likewise Babbit et al. ${ }^{11}$ observed improved regional myocardial blood flow and improved ventricular function in animals treated with intracoronary adenosine 3 hours after reperfusion following a 2-hour period of ischemia. In adjacent trans-
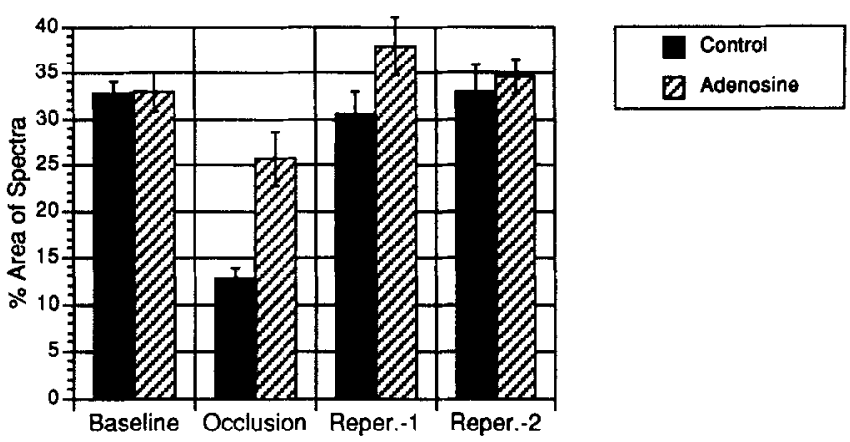

Fig. 5. Relative peak areas for phosphocreatine peaks, expressed as percentage of total peak area, are shown (mean $\pm \mathrm{SEM}$ ).

murally injured zones, there was evidence of reduced injury to the microvasculature. In a later study similar cardioprotective effects of intravenous adenosine on infarct size and ventricular function were seen after 90 minutes of occlusion ${ }^{17}$; however, in a third set of experiments involving a longer 3 -hour period of ischemia, no beneficial effects of adenosine administration on infarct size or regional wall function were observed. ${ }^{18}$ Thus the cardioprotective effects of adenosine may be dependent on the duration of the ischemic insult and may be limited by a lengthy period of severe ischemia. A similar cardioprotective effect has been reported in rabbits. In one experiment consisting of a 30-minute occlusion of an obtuse marginal branch of the left circumflex artery followed by a lengthy 48 -hour reperfusion period, adenosine administered during early reperfusion was shown to reduce infarct size in comparison with that in control animals. ${ }^{19}$

In a study by Hoffmeister et al., ${ }^{20}$ adenosine was infused via the intracoronary route in a series of dogs during a reperfusion period that followed a 45 minute period of coronary occlusion. The adenosine enhanced the repletion of high-energy phosphates in the tissues but interestingly did not improve ventricular function, suggesting that myocardial stunning may be related to other mechanisms. A number of experiments have been performed on isolated heart preparations; most ${ }^{21-24}$ but not all $^{25}$ document a beneficial effect for adenosine administration. A study by Bolling et al. ${ }^{24}$ involving isolated adult rabbit hearts showed that adenosine improved myocardial adenosine levels but not ATP levels during ischemia. During recovery the compound improved functional recovery and ATP levels. The differences between these results and ours may be a consequence of the substantial differences between isolated heart models and intact animals or may be due to the fact that 


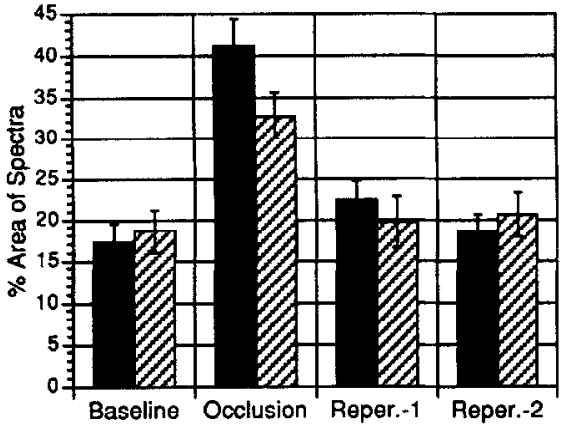

Fig. 6. Relative peak areas for inorganic phosphate peaks, expressed as percentage of total peak area, are shown (mean \pm SEM). See text for discussion of potential errors in determination of these peaks when they are small, that is, outside of occlusion period.

our surface coil sampled a larger area of myocardium than the biopsy specimens described in this report.

In most of the studies cited thus far, adenosine has been shown to have a beneficial effect on myocardial ATP levels, ventricular function, or both. In many of these studies, the agent is given after the ischemic insult; therefore, a mitigating effect of adenosine on reperfusion injury is suggested. Adenosine is believed to prevent cytotoxic changes through inhibition of neutrophil aggregation and adherence, neutrophilmediated generation and release of superoxide anion, and release of degradative enzymes including myeloperoxidase, $\beta$-glucuronidase, and lysozyme. ${ }^{14,26-29}$ These actions of adenosine suggest that one effect may be prevention of the inflammatory response once injury has taken place. In the studies of Olaffson et al. ${ }^{10}$ and Babbit et al., ${ }^{11}$ evidence of decreased infiltration of leukocytes in ischemic areas with attenuated cellular edema, preservation of the microvasculature, and a concomitant increase in blood flow was observed in ischemic areas after reperfusion. Thus a mechanism of cardioprotection was suggested to be the limitation of the deleterious changes associated with reperfusion injury and progression of the "noreflow" phenomenon.

In the present study no significant differences were observed between the treated and control groups during reperfusion. One possible explanation is the limited sensitivity of our measurement technique. Biological explanations for this failure include the fact that the reperfusion period in our study may have been too short for metabolic differences to be observed. Although it is generally believed that reperfusion injury begins with the hyperemic response immediately after the release of coronary occlusion, a longer reperfusion time than the one used in the present study ( 20 minutes) is likely needed for

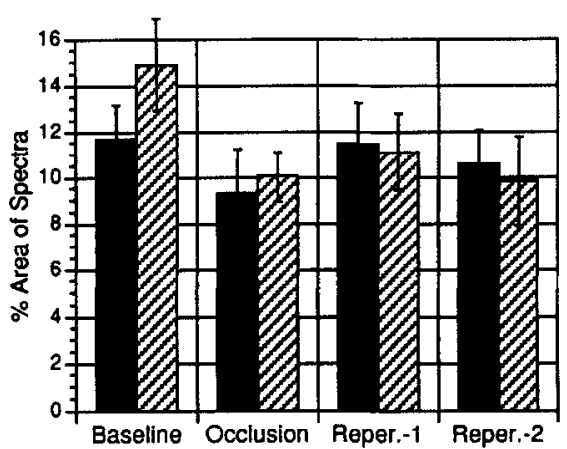

Contro

Q Adenosine

Fig. 7. Relative peak areas for ATP peaks, measured from $\beta$-peak and expressed as percentage of total peak area, are shown (mean \pm SEM).
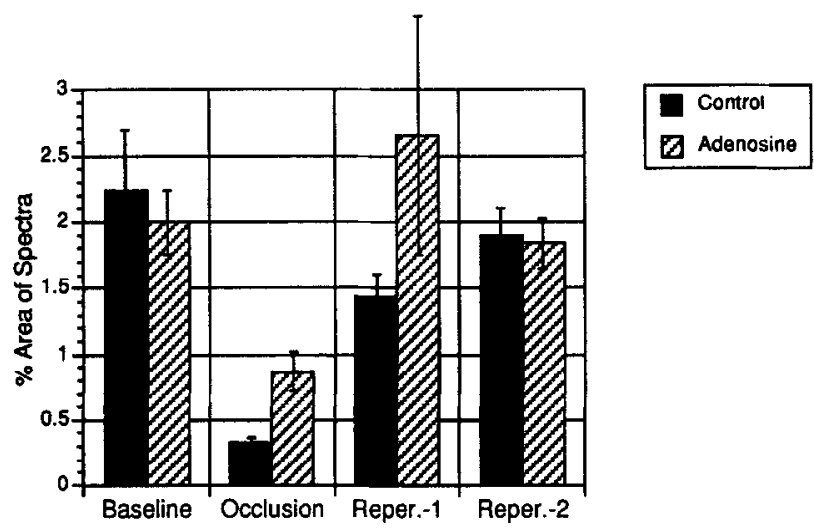

Fig. 8. Calculated peak area ratios of phosphocreatine over inorganic phosphate are shown (mean \pm SEM).

these changes to be observed. ${ }^{30-32}$ Differences in the length of ischemia might also explain the disparate results. We used a short period of ischemia (10 minutes). Although it is well accepted that a zone of ischemia progresses from subendocardium to subepicardium beginning after approximately 5 minutes of coronary occlusion in rabbits, subendocardial infarction is not produced until 15 minutes of occlusion. ${ }^{33}$ Long periods of ischemia, perhaps leading to more irreversible changes, may allow other benefits of adenosine to be observed during the reperfusion period.

Adenosine is known to be a potent dilator of coronary resistance vessels. ${ }^{13,31}$ One possible mechanism by which adenosine serves to protect high-energy phosphate metabolism during ischemia could be through improved coronary blood flow, either directly via dilation of resistance vessels or indirectly through augmentation of collateral flow; this mechanism was suggested by Ledingham et al. ${ }^{23}$ Enhanced oxygen delivery to ischemic areas could help main- 
tain the overall balance between oxygen supply and demand; however, in previous experiments in this laboratory, injection of microspheres demonstrated little or no myocardial blood flow to ischemic regions with the use of this intact rabbit model during coronary occlusion. 5

Another property of adenosine that may contribute to its cardioprotective effect is its antiadrenergic action. ${ }^{33-35}$ Antiadrenergic action may decrease myocardial oxygen demand during ischemia, thus contributing to preservation of high-energy phosphates. This negative inotropic and chronotropic effect offers one possible explanation for our findings. Rabbits treated with adenosine averaged a $20^{\circ}$ lower heart rate at baseline, during ischemia, and on reperfusion, suggesting a clear trend toward relative bradycardia.

Several studies have investigated a possible cardioprotective effect of adenosine arising from its role as a metabolic precursor of ATP. Mauser et al. ${ }^{36}$ demonstrated a 90 -fold increase in adenine nuclide de novo synthesis and increased tissue ATP levels with the administration of adenosine during reperfusion in postischemic myocardium in dogs. Results of experiments conducted by Foker et al. ${ }^{37}$ in dogs subjected to cardiopulmonary bypass also suggest that adenosine, when administered with an inhibitor of adenosine catabolism, enhanced the recovery of myocardial ATP levels after termination of the bypass. Consistent results have been obtained in another investigation through administration of adenosine deaminase, which catabolizes adenosine. Isolated hearts receiving this enzyme during ischemia showed decreased functional recovery and lower ATP levels, whereas hearts treated with adenosine showed increased function and higher ATP levels. ${ }^{38}$ Similarly, in a study of open-chest dogs, treatment with an inhibitor of adenosine catabolism protected contractile function after 15 minutes of ischemia. ${ }^{39}$

Maintaining levels of high-energy phosphates for normal cellular metabolic processes may play a role in cardioprotection, since these are energy-dependent processes that depend on adequate stores of ATP. Adenosine may either decrease degradation of ATP or enhance reconstitution of ATP via synthetic pathways, preserving the metabolic functions of the cell and limiting myocardial injury. In a recent study by Gruber et al.,40 using pretreatment with 5 -amino4 -imidazole carboxamide-riboside in intact animals to enhance concentrations of endogenous adenosine during ischemia, treatment groups showed an increase in endogenous adenosine only during net hydrolysis of ATP. ${ }^{40}$ Treatment groups were also found to have increased regional myocardial blood flow and fewer ventricular arrhythmias during ischemia, sug- gesting that treatment with agents that salvage high-energy phosphate synthesis before ischemia may have beneficial effects that extend beyond increasing energy stores for metabolic processes alone. Clearly, the full range of cardioprotective effects of adenosine has not yet been fully elucidated.

There has been at least one prior investigation in which ${ }^{31} \mathrm{P}$ NMR spectroscopy was used to characterize the effects of adenosine on ischemic myocardium. In an isolated guinea pig heart model, preservation of high-energy phosphates during ischemia, with accelerated resynthesis of ATP during several hours of reperfusion by means of treatment with adenosine, was observed. ${ }^{15}$ In this study, preservation of highenergy phosphates was associated with preserved myocardial function. Our own results are consistent with those of most previous investigations and are significant in that they confirm the enhanced highenergy phosphate levels in an intact animal model of regional ischemia. We observed the beneficial effects during the ischemic period, and there was also a trend toward improved high-energy phosphate levels during reperfusion. Intact animal models are clearly more physiologic than isolated heart preparations, and the real-time NMR technique is different from and hence complementary to biopsy techniques. The NMR method samples a larger area of myocardium, and measures both relative phosphocreatine and ATP levels, so there is no possibility that ATP degradation can be obscured between biopsy and assay.

The present study has several limitations. We only measured high-energy phosphate levels and did not assess ventricular function or the size of the zone of ischemic myocardium. Some but not all of the previously cited investigations show discordance between high-energy phosphate levels and ventricular function. It is also possible that our ${ }^{31} \mathrm{P}$ NMR imaging technique, which required several minutes of acquisition time, was not adequately sensitive to detect small or transient changes in high-energy phosphate levels during a brief period of reperfusion. Moreover, there are well-known difficulties with this model related to the signal-to-noise ratio, which may make it difficult to detect smaller changes. Although the gross effects of coronary occlusion were observed on release of coronary occlusion, it would have been helpful to look at the histologic and biochemical evidence of reperfusion injury after a brief period of ischemia. The addition of biochemical markers of neutrophil activity might have added to the sensitivity of this model in yielding insights into the cardioprotective effects of adenosine and various interventions during a brief period of ischemia. 
Several known limitations of magnetic resonance methodology should be stated briefly, inasmuch as they influence the interpretation of the quantitative data. We did not perform a saturation correction on the peak areas; hence relative peak areas of different metabolites as reported here will not be equally proportional to the respective metabolite concentrations. Thus our ratios may differ from the corrected results reported by others. There is substantial overlap hetween spectral peaks, particularly in the crucial inorganic phosphate region. There may also be spec. tral contamination of the inorganic phosphate resonance by $2,3-\mathrm{DPG}$ in the blood. These factors will reduce the accuracy of our inorganic phosphate measurements. The spectral phasing, baseline correction, and peak fitting techniques used have known limitations as well. ${ }^{41}$ Spectral localization was achieved by the proximity of the myocardium to the surface coil; thus, partial volume effects between ischemic and nonischemic myocardium are possible. Differences in high-energy phosphate levels may reflect both differences in tissue levels in ischemic myocardium and differences in the size of the ischemic zone. The object of these experiments was to detect differences between adenosine-treated and control groups. Inasmuch as the same acquisition and analysis parameters were used in both groups, these limitations are not expected to change the validity of the intergroup comparisons.

This study did not address the long-term effects of a brief period of ischemia or the long-term efficacy of adenosine. Since changes in phosphocreatine levels of borderline significance during early reperfusion were noted in rabbits treated with adenosine, it is possible that additional studies utilizing longer periods of reperfusion would detect significant differences in high-energy phosphate levels during reperfusion. In conclusion, we used NMR spectroscopy to compare myocardial high-energy phosphate metabolites in regionally ischemic myocardium in rabbits with and without treatment with adenosine. Adenosine preserved high-energy phosphate levels during severe ischemia but in our in vivo model did not alter high-energy phosphate levels during early reperfusion. Our metabolic data suggest that adenosine intervention during myocardial ischemia may be cardioprotective and limit ischemic injury.

We thank the General Electric Company for their support of the CSI facility.

\section{REFERENCES}

1. Jennings RB, Hawkins HK, Lowe JE, Klotman S, Reimer KA, Hill ML. Relation between high-energy phosphates and myocardial ischemia in the dog. Am J Pathol 1978;92:187-214.

2. Reimer KA, Hill ML, Jennings RB. Prolonged depletion of the adenine nucleotide pool due to delayed resynthesis of adenine nucleotides following reversible myocardial ischemic injury in dogs. J Mol Cell Cardiol 1981;13:229-39.

3. Bolli R, Zhu WX, Thornby JI, et al. Time course and determinants of recovery of function after reversible ischemia in conscious dogs. Am J Physiol 1988;254:H102-14.

4. Rehr RB, Tatum JL, Hirsch JI, Quint R, Clarke G. Reperfused-viable and reperfused-infarcted myocardium: differentiation with in vivo P-31 MR spectroscopy. Radiology 1989; 172:53-8.

5. Kavanaugh KM, Aisen AM, Fechner KP, Chenevert TL, Dunham WR, Buda AJ. Regional metabolism during coronary artery occlusion, reperfusion, and reocclusion using phosphorus $^{31}$ nuclear magnetic resonance spectroscopy in the intact rabbit. AM HEART J 1989;117:53-9.

6. Stein $\mathrm{PD}$, Goldstein $\mathrm{S}$, Sabbah $\mathrm{HN}$, et al. In vivo evaluation of intracellular $\mathrm{pH}$ and high energy phosphate metabolites during regional myocardial ischemia in cats using $31 \mathrm{P}$ nuclear magnetic resonance. Magn Reson Med 1986;3:262-9.

7. Wendland MF, White RD, Derugin N, et al. Characterization of high-energy phosphate compounds during reperfusion of the irreversibly injured myocardium using 31P MRS. Magn Reson Med 1988;7:172-83.

8. Nunnally RL, Bottomley PA. Assessment of pharmacological treatment of myocardial infarction by phosphorus-31 NMR with surface coils. Science 1981;211:177-80.

9. Wroblewski LC, Aisen AM, Swanson SD, Buda AJ. Evaluation of myocardial viability following ischemic and reperfusion injury using phosphorus 31 nuclear magnetic resonance spectroscopy in vivo. Am HFarT .J 1990;120:31-9

10. Olaffson B, Forman MB, Pruett D, Pou A, Cates CU, et al Reduction of reperfusion injury in the canine preparation by adenosinc: importance of the endothelium and the no-reflow phenomenon. Circulation 1987;76:1135-45.

11. Babbitt DG, Virmani N, Forman MB. Intracoronary adenosine administered after reperfusion limits vascular injury after prolonged ischemia in the canine model. Circulation 1989;80:1388-99.

12. Hoffmeister JW, Hoff P'I', F'letcher DD, Lucchesi BR. Combined adenosine and lidocaine administration limits myocardial reperfusion injury. Circulation 1990;82:595-608.

13. Braunwald E, Sobel BE. Coronary blood flow and myocardial ischemia. In: Braunwald $\mathrm{E}$, ed. Heart disease: A textbook of cardiovascular medicine. 3rd ed. Philadelphia: WR Saunders. 1988:1198-9.

14. Cronstein BN, Levin RI, Belanoff J, Weissman G, Hirschorn $R$. Adenosine: an endogenous inhibitor of neutrophil-mediated injury to endothelial cells. J Clin Invest 1986;78:760-70.

15. Yoshimaya M, Sakai M, Teragaki M, Takeuch K, Takeda T, Ikata M, Ishikawa M, Miura I. 31P-MRS study of bio-energy recovering phenomenon. Biochem Biophys Res Commun 1988;151:865-71.

16. Garlick PB, Radda GK, Seeley PJ. Studies of acidosis in the ischemic heart by phosphorus nuclear magnetic resonance. Biochem J 1979;184:547-54.

17. Pitarys II CJ, Virmani R, Vildibill Jr HD, Jackson EK, Forman MB. Reduction of myocardial reperfusion injury by intravenous adenosine administered during the early reperfusion period. Circulation 1991;83:237-47.

18. Babbitt DG, Virmani R, Vildibill Jr HD, Norton ED, Forman $\mathrm{MB}$. Intracoronary adenosine administration during reperfusion following 3 hours of ischemia: effects on infarct size, ventricular function, and regional myocardial blood flow. AM HEART J 1990;120:808-18.

19. Norton ED, Jackson EK, Virmani R, Forman MB. Effect of intravenous adenosine on myocardial reperfusion injury in a model with low myocardial collateral blood flow. AM IIEART J 1991;122:1283-91.

20. Hoffmeister HM, Mauser M, Schaper W. Effect of adenosine and AICAR on ATP content and regional contractile function in reperfused canine myocardium. Basic Res Cardiol $1985 ; 80: 445-58$. 
21. Angello DA, Headrick JP, Coddington NM, Berne RM. Ade osine antagonism decreases metabolic but not functir recovery. Am J Physiol 1991;260:H193-200.

22. Bolling SF, Bies LE, Gallagher KP, Bove EL. Enhanc ocardial protection with adenosine. Ann Thorac Surg 1 yuy; 47:809-15.

23. Ledingham S, Katayama O, Lachno D, Patel N, Yacoub M. Beneficial effect of adenosine during reperfusion following prolonged cardioplegic arrest. Cardiovasc Res 1990:24:247-53.

24. Bolling SF, Bies LE, Bove EL, Gallagher KP. Augmenting intracellular adenosine improves myocardial recovery. J Surg Res 1991;50:629-33.

25. Hohlfeld T, Hearse DJ, Yellon DM, Isselhard W. Adenosineinduced increase in myocardial ATP: are there beneficial effects for the ischemic myocardium? Basic Res Cardiol 1989; 84:499-509

26. Cronstein BN, Kramer SB, Weissman G, Hirschhorn R. Adenosine: a physiologic modulator of superoxide anion generation by human neutrophils. J Exp Med 1983;158:1160-77.

27. Engler R. Consequences of activation and adenosine-mediated inhibition of granulocytes during myocardial ischemia. Fed Proc 1987;46:2407-12.

28. Roberts PA, Newby AC, Hallett MB, Campbell AK. Inhibition by adenosine of reactive oxygen metabolite production by human polymorphonuclear leukocytes. Biochem J 1985;277:66974.

29. Nielson CP, Vestal RE. Effects of adenosine on polymorphonuclear leukocyte function, cyclic $3^{\prime} 5^{\prime}$-adenosine monophosphate, and intracellular calcium. Br J Pharmacol 1989;97: $882-8$.

30. Andreoli SP. Mechanism of endothelial cell ATP depletion after oxidant injury. Pediatr Res 1989;25:97-101.

31. Hernandez LA, Grisham MB, Twohig B, Arfors KE, Harlan JM, Granger DN. Role of neutrophils in ischemic reperfusion and microvascular injury. Am J Physiol 1987;253:H699-704. Dobson JG. Adenosine reduces catecholamine contractile responses in oxygenated and hypoxic atria. Am J Physiol 1983;245(Heart Circ Physiol 14):H475-80.

Connelly C, Vogel WM, Hernandex YM, Apstein CS. Movement of necrotic wavefront after coronary artery occlusion in rabbit. Am J Physiol 1982;243:H682-90.

i4. Dobson JG, Ordway RW, Fenton RA. Endogenous adenosine inhibits catecholamine contractile responses in normoxic hearts. Am J Physiol 1986;25:H455-62.

35. Dobson JG. Mechanism of adenosine inhibition of catecholamine induced responses in the heart. Circ Res 1983;52:151-60.

36. Mauser M, Hoffmeister HM, Nienaber C, Schaper W. Influence of ribose, adenosine and "AICAR" on the rate of myocardial adenosine triphosphate synthesis during reperfusion and coronary occlusion in the dog. Circ Res 1985;56:22030.

77. Foker JE, Einzig S, Wang T. Adenosine metabolism and myocardial preservation: consequences of adenosine catabolism on myocardial high-energy compounds and tissue blood flow. J Thorac Cardiovasc Surg 1980;506-16.

18. Bolling SF, Bove EL, Gallagher KP. ATT precursor depletion and postischemic myocardial recovery. J Surg Res 1991;50:629. 33.

9. Koke JR, Fu LM, Sun D, Vaughan DM, Bittar N. Inhibitors of adenosine catabolism improve recovery of dog myocardium after ischemia. Mol Cell Biochem 1989;86:107-13.

0. Gruber HE, Hoffer ME, McAllister DR, Laikind PK, Lane TA Schmid-Schoenbein GW, Engler RL. Increased adenosine concentration in blood from ischemic myocardium by AICA riboside: effects on flow, granulocytes and injury. Circulation 1989;80:1400-11.

1. Bottomley PA. The trouble with spectroscopy papers. Radiology 1991;181:344-50. 